\title{
EDICIÓN DIGITAL Y ELECTRÓNICA EN ESPAÑA: UN ESTADO DE LA CUESTIÓN
}

\author{
Julio Peiró Sempere \\ Mireya Fernández Merino \\ Elena Martínez Carro ${ }^{* * *}$
}

\begin{abstract}
RESUMEN: El presente trabajo reflexiona acerca de los retos que el sector editorial en España debe afrontar para adecuar su oferta a las nuevas necesidades y expectativas generadas por los lectoresusuarios en la actual era digital. Partiendo de un cambio en el concepto de textualidad propiciado por los nuevos soportes de lectura, se hace hincapié en las novedades que las ediciones críticas en formato digital deberán aportar para acercar los nuevos contenidos a un público especializado que demanda cada vez más una mayor interacción con el texto y los soportes de lectura. Asimismo, se destaca la importancia de aunar esfuerzos entre los profesionales en el ámbito de la edición textual, la filología y la informática para llegar a un consenso que ponga fin al "caos" reinante en el mundo de la edición crítica digital.
\end{abstract}

PALABRAS CLAVE: Edición crítica digital. Bibliotecas virtuales. Lenguajes de marcación. Hipertextualidad. Usuario-lector.

\section{Introducción}

Afirma Andrew Piper en su revelador libro Book was there: Reading in Electronic Times (2012) que puede imaginar un mundo sin libros, pero no un mundo sin lectura. $Y$ es que abordar las repercusiones que el actual auge de la edición electrónica vive en España supone, en primer lugar, poner el centro de atención en la figura emergente de un nuevo lector, en su relación con el texto y los dispositivos que lo almacenan $y$, finalmente, en lo que bien podría denominarse en términos comerciales como experiencia de usuario final. La batalla por captar nuevos lectores en la era digital ha favorecido la proliferación de dispositivos electrónicos de lectura, la acumulación de títulos digitalizados en los catálogos de las editoriales y, cómo no, la necesidad de replantearse y tener en cuenta las necesidades o expectativas que este tipo de lector ha depositado en la experiencia lectora. En este sentido, afirma

\footnotetext{
“Universidad Internacional de La Rioja, España. E-mail: julio.peiro@unir.net

* Universidad Internacional de La Rioja, España. E-mail: Mireya.fernandez@unir.net

${ }^{* * *}$ Universidad Internacional de La Rioja, España. E-mail: elena.martinez@unir.net
} 
Lucía Megías (2010) que las novedades o los cambios en el proceso de lectura vienen dados por un nuevo "soporte de lectura y difusión de los textos". Hablamos por tanto de una forma diferente de lectura influida por el medio de transmisión y las posibilidades que este medio digital ofrece. La evolución de la edición electrónica de textos deberá por tanto tener en cuenta los modernos hábitos de consumo. Desde la inmediatez en la adquisición de la obra, pasando por la interacción con el propio texto o la búsqueda de soluciones a la necesidad que muchos lectores tienen de compartir opiniones o incluso el propio texto, la industria editorial aborda en la actualidad retos propios de una revolución, la que vivimos en la actual era digital.

La necesidad de compartir opinión e información en un mundo digital dominado por las redes sociales obliga en cierto modo a reflexionar sobre la forma en la que compatibilizar esta exigencia por compartir el texto, su contenido y las opiniones sobre él, con el papel de la autoría y los derechos de autor. Sin embargo, es el usuario-lector el que reclama estas novedades y es él el que debe ser preguntado. Como ejemplo, nos gustaría tomar en consideración un breve trabajo sobre los hábitos de lectura realizado entre 123 alumnos de la Universidad Internacional de La Rioja en 2014 (Peiró, 2014), donde nos llamó poderosamente la atención la respuesta de un alumno a la pregunta sobre qué se puede hacer con un libro impreso y no con una edición digital del mismo. En su respuesta, el alumno argumentaba que no podía prestar o regalar dichas ediciones digitales a una tercera persona. En definitiva, este cambio en el soporte de lectura condiciona de manera notable la experiencia que el usuario o lector obtiene. Si bien el aspecto sensorial del proceso, el poder tocar el libro, olerlo, manipularlo como si de un objeto de culto se tratase, se ve mermado en la edición electrónica, muchos otros aspectos novedosos pueden incorporarse en esta nueva forma de leer. Es aquí donde la edición electrónica tiene sus retos. Se trata por tanto de cuestiones que deberán ser abordadas en este emergente mundo de la edición electrónica y sus posibilidades de difusión. Siguiendo con los cambios en la difusión del texto, argumenta Lucía Megías (2010) que esta nueva "difusión digital" obliga a replantearnos el propio concepto de "textualidad" y sus implicaciones en el mundo de la cultura y, por supuesto, en el ámbito de las denominadas Humanidades Digitales. En este sentido, el interés por 
esta disciplina y el conjunto de prácticas que reúne no es nuevo en España. Desde finales de los 80 y comienzos de la década de los 90 la relación literatura, informática y tecnología digital ha sido objeto de reflexión por parte de los humanistas. Las voces de alerta acerca del futuro del libro y de la crítica textual en un mundo dominado por el avance tecnológico e informático han encontrado eco en esa parcela de la academia dedicada al estudio de la literatura y de manera especial a la filología clásica. Así, las actuales generaciones que crecen bajo la sombra de nuevos y cambiantes dispositivos electrónicos obligan a los académicos a interrogarse sobre las formas de acceder al saber, las nuevas prácticas de lectura, la creación y transmisión del conocimiento, la expansión y mejora de los métodos de interpretación en una sociedad asistida por los ordenadores (Price y Siemens, 2013).

La premisa de que la tecnología computacional puede contribuir a alcanzar las metas tradicionales a las que aspiran las humanidades ha orientado el trabajo de muchos filólogos comprometidos en demostrar cómo los nuevos instrumentos pueden enriquecer el trabajo crítico. Sin embargo, los esfuerzos de más de cinco décadas desde que Roberto de Busa emprendiera en 1949 su Index Thomisticus y abriera el camino a las ediciones digitales no borran las inquietudes que persisten acerca del futuro de la edición crítica y de la capacidad de los investigadores para integrar en el hacer filológico los instrumentos digitales desarrollados en este campo. A esto se suma el complejo laberinto de procesos socioculturales, históricos y geográficos relacionados con las prácticas filológicas, fuerzas que inciden directamente en la triada integrada por la editorial, el profesional a cargo de la edición y el lector, cuyo resultado es un producto específico: el texto. Algunas de estas fuerzas que orientan el cambio del texto tradicional a su versión digital han sido objeto de reflexión: el desarrollo exponencial de la producción de documentos y su fácil acceso; el surgimiento de instrumentos específicos que facilitan el trabajo académico; las nuevas políticas de edición; el papel de los lectores, sus expectativas y posibilidades como actores-usuarios que organizan sus propio itinerario de lectura (Apollon, Bélisle y Régnier, 2014). 
Los académicos de las universidades españolas han buscado respuesta a estas inquietudes que despierta el renovado y bienvenido diálogo entre las Humanidades y las Ciencias. Una visión panorámica sobre el estado de las Humanidades Digitales en nuestro país revela el interés de los especialistas por comprender, en un primer momento, los antecedentes de la era digital, el análisis de la relación entre el libro y el hipertexto, los cambios que el uso del ordenador introduce en la manera de interactuar con los documentos, así como los principales proyectos de digitalización realizados desde mediados de los años ochenta del pasado siglo, su evolución en España a partir de la primera década de este segundo milenio, aspecto que se consolida con la realización del primer congreso oficial sobre las Humanidades Digitales en 2013 (Rojas Castro, 2013).

Asimismo, la reflexión académica se centra en la necesidad de sumar ventajas a la edición digital, trascender ese primer momento en que la facilidad de acceso a los textos en los diferentes formatos digitales, sea complementada por nuevas ofertas que brinden al lector, por ejemplo, la posibilidad de leer y comparar versiones de una obra y juzgar las decisiones editoriales; de utilizar enlaces que conecten los textos con documentos críticos o recursos multimedia que enriquezcan la comprensión de las obras; el diseño de una infraestructura informática que haga posible recuperar información textual mediante búsquedas estructuradas (Rojas Castro, 2013).

La preocupación común ha tomado, sin embargo, rutas diferentes en función del interés de los investigadores. El estudio de la edición electrónica corre en paralelo al interés en la edición crítica. El imperativo: precisar términos, analizar los logros alcanzados y sus limitaciones. Numerosos son los trabajos. El amplio panorama impone la selección y a partir de ella el análisis de los puntos que acaparan el interés de los investigadores.

\section{La edición crítica digital}

El interés por difundir los fondos de las bibliotecas o los textos clásicos y facilitar su análisis e interpretación a los expertos ha impulsado el trabajo de la edición crítica 
digital. Algunas de las sendas recorridas no han sido diferentes a las transitadas en el mundo editorial. En un primer momento, los textos pasan al medio electrónico manteniendo, en la medida de lo posible, las características del libro analógico. Un paso adelante lo constituye la adaptación al nuevo entorno al hacer uso de las ventajas tecnológicas que permiten interrelacionar texto, imagen y sonido. Se pasa de una relación especular a una relación emergente (Rodríguez de las Heras, 2001). Surge así un nuevo paradigma, la búsqueda de un modelo hipertextual que convierta la información -esté dada en palabras, en imágenes o en sonidos-en una estructura de unidades textuales enlazadas dentro de un mismo documento o con otros externos, que requieren la participación activa del lector para su lectura y utilización (Pajares, 2004). Una forma de creación, de difusión del saber y también de edición. Interesan en nuestro caso los dos últimos aspectos que inciden en la forma cómo se han editado y difundido los textos literarios en la red, cómo se han ampliado los proyectos con el avance de programas y herramientas informáticas, hasta dar forma a lo que se ha dado en denominar "el incunable del hipertexto" (Lucía Megías, 2008a). Los logros alcanzados hasta el presente alimentan la imaginación y abren la posibilidad de diseñar nuevos modelos de edición crítica cuyo objetivo esté al servicio del conocimiento.

La promoción de ediciones digitales en España recoge el esfuerzo de bibliotecarios, archiveros y especialistas dedicados al estudio de textos raros y modernos. Surgen las bibliotecas digitales, nombre genérico que designa un modelo de almacenamiento que incluye documentos en formato analógico digitalizados para facilitar a los usuarios su consulta, o documentos creados ex profeso de manera digital, cuya finalidad es la divulgación y accesibilidad de sus materiales, y que responden a tres aspectos básicos: la acumulación de material, la necesidad de organizarlo internamente, y la capacidad de recuperarlo con un sistema de búsqueda amable al usuario. De acuerdo a su interés y al tipo de fondos, las bibliotecas han dedicado sus esfuerzos a reproducir: en facsímil testimonios manuscritos o impresos, libros raros o antiguos; textos difundidos previamente en formato analógico; textos digitalizados originalmente; hipertextos. 
En el primer caso, encontramos el trabajo realizado por bibliotecas como la Biblioteca Digital Hispánica, de la Biblioteca Nacional de España (bdh.bne.es), la Biblioteca de Catalunya (http://www.bnc.cat), la Biblioteca Digital Dioscórides, de la Universidad Complutense de Madrid (biblioteca.ucm.es/atencion/24063.php) cuyo interés se ha centrado en la digitalización de parte de sus documentos más valiosos para cumplir con el objetivo de facilitar a los investigadores sus fondos sin acudir obligatoriamente a sus instalaciones. En otros casos, se reúne, además, la documentación de un conjunto de ellas, como ocurre en el caso de la Biblioteca Digital Valenciana (vivaldi.gva.es). También es posible acceder a los bienes documentales de instituciones que comparten un interés común; tal es el caso de la Biblioteca Joan Lluís Vives (http://www.lluisvives.com), que recoge un número significativo de testimonios antiguos de los clásicos de la literatura catalana.

El segundo grupo, la digitalización de ediciones analógicas dedicadas a la transcripción crítica de un testimonio o de ediciones críticas de un texto, corresponde con la mayor parte de los documentos que pueden consultarse en las bibliotecas. La calidad textual de las ediciones en estos casos varía. El volumen y la difusión ha sido el objetivo principal que no encuentra equivalencia en otro criterio necesario al momento de convertir un texto analógico en digital: el criterio de autoridad. Se omite, en la mayoría de los casos, la identidad de la edición de base para la digitalización o, en caso de especificarse, su justificación carece de base científica. Este aspecto, característico de las primeras iniciativas, se ha ido superando al incorporarse ediciones en las que se ha tomado en cuenta el rigor filológico. Es el caso de la Biblioteca Miguel de Cervantes (www.cervantesvirtual.com), el proyecto de mayor envergadura realizado en España, en el que es posible rastrear ambas tendencias: la acumulación de material y los avances alcanzados con el transcurso de los años, aspecto representado en las diferentes áreas que se encuentran en ella (Bibliotecas española, americana, Joan Lluís Vives, das Letras Galegas, de Literatura Infantil y Juvenil, de Signos, Historia, Hemeroteca) cuya lista ha aumentado acompañada de mejoras en la calidad de edición. 
El tercer ejemplo, la digitalización de textos que han sido creados de manera expresa para un portal, se encuentra en Parnaseo, proyecto de la Universidad de Valencia (http://parnaseo.uv.es) dedicado a la literatura medieval y renacentista, e incluye un material diverso que recoge ediciones digitales de textos, reproducciones facsímiles, transcripciones y ediciones críticas, revistas, editorial, enlaces a otras bibliotecas y bases de datos. Desde su creación en 1996 hasta el presente ha aumentado su patrimonio de ediciones inéditas de textos hispánicos a cargo de especialistas a lo que se suma las ventajas de herramientas informáticas que facilitan el trabajo del lector.

Por último, es posible encontrar otros proyectos que si bien parten de las propuestas editoriales del mundo analógico, tienen como objetivo el diseño de nuevos modelos editoriales. Se incluyen en este caso las ediciones genéticas y las ediciones sinópticas integrales. Un ejemplo es el Portal de textos Múltiples Andrés de Poza (andresdepoza.com) que, como se explica en su página de presentación, edita una colección de obras señeras de la literatura religiosa o política europea de los siglos $\mathrm{XVI}$ y XVII en las que se presenta el original junto a traducciones a otras lenguas, además de otros textos no literarios -documentos fundacionales o confirmaciones de derechos o privilegios- que incluye bajo el concepto de textos notariales. Otros proyectos como la Edición variorum electrónica del Quijote (http://www.csdl.tamu.edu/cervantes/V2/variorum/index.htm) o Cibertextos, desde hace varios años apoyado por la Biblioteca Virtual Miguel de Cervantes, representan otro avance en el camino transitado por las bibliotecas virtuales hipertextuales, donde el usuario encuentra, además de las ediciones, un conjunto de herramientas informáticas y materiales que enriquecen el portal.

El seguimiento y análisis de estos proyectos (Lucía Megías, 2008a, 2008b, 2009) muestra que el interés por aprovechar las ventajas de difusión del nuevo medio influyó en que prevaleciera el criterio de cantidad -más textos digitalizados- y el económico -no tener que pagar los derechos de autor- en aquellos proyectos que con éxito se emprendieron desde la década de los años noventa del pasado siglo. Así, el trabajo de las bibliotecas virtuales en un primer momento, con determinadas 
excepciones, se desarrolla bajo la premisa de acumulación. Interesaba almacenar la información, la cantidad de textos incorporados, ciertamente representativo del esfuerzo dedicado a esta tarea; pero los errores cometidos: la heterogeneidad de la procedencia de las ediciones digitalizadas o la carencia de información sobre los criterios que las sustentaban restaron valor al esfuerzo realizado. La calidad filológica quedaba así relegada a un segundo plano. A esto se sumaba la dificultad para acceder al contenido de la información y la visualización de los resultados.

El cambio en los últimos años -como puede constatarse al consultar algunos de los portales de la Biblioteca Virtual Cervantes- representa la inclusión del rigor científico en la selección y organización del material, alcanzado en el momento en que se reconoce el papel de los especialistas, los filólogos, quienes conocen y evalúan los materiales que se han de incorporar y la estructura que ha de tener la información en la web. Especial atención merece la inclusión de los criterios de selección así como la orientación metodológica sobre la que se construye y estructura el material. Es el caso por ejemplo del portal de Andrés Pozas en el que el lector, al pulsar el cursor sobre el apartado "El proyecto", encontrará desplegado los objetivos y las metodologías de las ediciones, del alineamiento y de las correspondencias léxicas (andresdepozas.com/el-proyecto). En este sentido, las mejoras incluyen el enriquecimiento de la información que ofrecen los portales sobre una obra o un autor, pues se difunden el texto crítico, variantes de los manuscritos que han transmitido un determinado texto o las variantes de otras ediciones. A esto se suma la inclusión de un mayor número de herramientas informáticas para la consulta del texto: buscadores textuales, concordancias, índices y estadísticas, y la mejora en la visualización de los materiales -todavía bajo la influencia del libro de papel y la lectura tradicional- que incorpora colores y tipos de letra como portadores de información, la posibilidad de realizar búsquedas en el interior de los textos y testimonios (http://aaswebsv.aas.duke.edu/celestina).

Cabe destacar por tanto que las ediciones críticas, dependiendo de la naturaleza de la obra objeto de la edición, han incluido materiales diversos que van desde facsímiles, pasando por el texto crítico, el aparato de variantes, el conjunto de notas 
críticas, hasta incluir bibliografía, apéndices o material de apoyo (visual, acústico, textual). Su presentación en el mundo digital ha traspasado los límites del formato analógico y creado "el incunable del hipertexto". Los desafíos que conllevan los proyectos de digitalización imponen la creación de modelos hipertextuales de difusión, en diálogo con el saber filológico y las necesidades del usuario. ¿Cuáles son los retos?

\section{EI horizonte futuro}

Las consecuencias de este nuevo paradigma textual quizá deriven en nuevas y diversas teorías de la lectura digital, o en nuevas fórmulas de edición, distribución y comercialización de estos textos que todavía hoy no podemos vislumbrar. No se trata por tanto de disponer de un texto en un formato digital, sea el que sea, como una alternativa a la edición impresa de la misma obra. Este nuevo lector en la era digital espera o anticipa una experiencia diferente al sentarse frente a su dispositivo electrónico de lectura. La interacción, la posibilidad de acceder a nuevos contenidos, o la experiencia lectora compartida en tiempo real son solo algunas de las posibilidades abiertas. Con todo esto, la edición electrónica de textos cumple un papel formidable en la adaptación de los contenidos a los distintos tipos de lectores, o usuarios. En este sentido, tal y como apuntan los miembros de la asociación "Laboratorio de ideas sobre el libro" en su trabajo titulado La gran transformación: panorama del sector del libro en España (2012-2015), el papel del editor sigue siendo fundamental en la actualidad para ofrecer el producto adecuado en función del tipo de usuario o lector. Así, esta obra ofrece claves imprescindibles para no perderse en el inminente futuro de la edición electrónica en España. En ella se nos muestra el panorama que está por venir y que requiere de una revisión de los métodos tradicionales de edición y distribución del libro para adecuarse a las nuevas exigencias o expectativas creadas en este mundo digital de la información y el contenido. Aspectos como la autoedición, el marco regulatorio y los derechos de autor, los planes de fomento de la lectura o el papel de las librerías y la crítica en la promoción y comercialización de las obras son solo algunos de los aspectos claves tratados. $\mathrm{Y}$ es que el panorama editorial actual plantea, como hemos mencionado, 
numerosos interrogantes, como la posibilidad de ofrecer ediciones en función de la demanda de los consumidores. Como afirma Lucía Megías (2010, p. 248) "se trata de que el usuario -previamente registrado- tenga la posibilidad de personalizar la edición electrónica [...]".

La edición electrónica se encuentra en estos momentos en una encrucijada por los avances hechos, pero también por el ingente material volcado en la red con características diferentes y calidades desiguales. Los materiales son sumamente diversos y con fines parciales. Podemos afirmar -sin temor a equivocarnos- que el lector medio se encuentra ante un laberinto de difícil accesibilidad. El libro desde las plataformas digitales o desde las bibliotecas virtuales hipertextuales ha dejado atrás al mayor número de lectores. Solo el libro electrónico, de formato cerrado (e-pub), se extiende como competidor del libro tradicional. Hasta el momento son muy pocos los experimentos hipertextuales que desde la edición crítica han dado un salto a la "popularidad". Los lectores siguen prefiriendo el formato de papel al digital para las obras clásicas y las experiencias de la crítica textual en la red tienen poco sentido para el lector medio. El libro digital ha encontrado "su hueco" en la divulgación y se considera útil por su portabilidad y economía. De ahí que se haya asimilado al libro de distracción o a los best seller. Lo que hace una década parecía ser "el fin del libro" -como se ha definido desde su creación- por la aparición del formato digital, ha dejado de constituir una amenaza en las proporciones que se profetizaban.

Sin embargo, no cabe duda de que poco a poco el libro electrónico ganará fuerza especialmente con la llegada de los nativos digitales, aunque no son menos ciertos los retos que se presentan a la edición digital y que tratamos de resumir en los siguientes puntos.

1. Ordenar el caos. Los veinte últimos años de edición digital han permitido que se vuelquen todo tipo de textos en formatos y plataformas muy diversas entre sí, que a su vez han trabajado desde métodos diferentes y al hilo de programas informáticos que se iban creando según las necesidades. Muchos de estos materiales han quedado obsoletos al mismo tiempo que conviven en la red con otros 
permanentemente actualizados y de indudable interés. La red se ha convertido en un "depósito" que "engulle" por igual lo deleznable y lo sublime, sin ofrecer al lector medio la seguridad de una calidad. Verdaderamente nos encontramos ante una "torre de Babel", como han señalado numerosos estudiosos. En una biblioteca física es fácil distinguir entre las ediciones antiguas, las autorizadas o divulgativas. Para su selección funcionan elementos de índole verbal y no verbal que el receptor conoce como referentes, y que condicionan la elección de un libro. Por el momento estos referentes son inexistentes en el complejo laberinto de la red. Se hace necesario como proyección de futuro para ordenar el ingente material que hay y que habrá-, construir plataformas que unifiquen los distintos materiales a manera de "lanzaderas" a las páginas más específicas. Es imprescindible que los diversos materiales de calidad se ordenen en portales generados desde "supraestructuras". Las grandes compañías de internet -como por ejemplo Google-, en comunión con instituciones investigadoras y universitarias, deben tomar esta iniciativa como ya lo han hecho en otros aspectos. Sin estas plataformas de acceso y de ordenación será difícil conseguir poner orden en el bosque actual de las ediciones digitales y en breve espacio de tiempo se hará necesario dedicar ingentes esfuerzos y tiempo para encontrar las ediciones digitalizadas de una obra como si de ejemplares pretéritos se tratara.

2. Distinguir el público. Por otro lado, la red constituye el elemento globalizador "per se". Su propia esencia es compartir el conocimiento entre todos. Las élites intelectuales quedan desterradas en el sistema de las tres www. Sin embargo, desde la perspectiva de la edición crítica, cabe preguntarse hasta qué punto este tipo de publicaciones ha interesado e interesa al gran público. La edición digital se encuentra todavía en una fase muy incipiente y el desconcierto ante las nuevas tecnologías y su integración en las disciplinas clásicas condicionan el objetivo primordial: la edición y accesibilidad de los textos. Es imprescindible conciliar ambos mundos sin olvidar al lector. El diálogo entre expertos de las dos orillas, y la necesidad de crear materiales que contemplen sendas perspectivas al servicio del lector, son un reto de futuro prioritario. Se impone por tanto la prioridad de distinguir distintos tipos de lector $-\mathrm{y}$ sus necesidades- para adecuar las ediciones a los gustos 
del público y no a la inversa. La conciliación entre el tipo de lector y el formato adecuado a la lectura que se quiere realizar es uno de los principales retos a los que se debe atender -no solo el sector comercial editorial- sino también los editores y técnicos.

3. Trascender la lectura analógica. No cabe duda de que el libro digital sigue anclado en trasladar los moldes tradicionales a los formatos electrónicos. Esta réplica ayuda a que muchos lectores vean en el libro digital una reproducción de los formatos que tenían asimilados. Sin embargo, esta lectura analógica está frenando otras formas de expresión digital que por el momento no se están incorporando a los dispositivos móviles. Generalmente los e-books admiten pocos formatos y las ediciones se hacen tan solo sobre los textos base, dejando a un lado las notas a pie de página o aclaraciones del editor, tan solo accesibles mediante links. La consulta de estas aclaraciones resulta un tanto difícil por lo que se acaba por omitir. Si siempre las notas a pie de página han sido arduas de leer, en el formato electrónico resultan más complejas. Hasta el momento son pocos los modelos de ediciones críticas entendidas como tal- que se ofrezcan desde el libro electrónico. Las causas son muy diversas, pero fundamentalmente se apunta hacia la falta de adecuación entre el formato de e-book y el avance de las tecnologías aplicadas a la edición. Son numerosos los esfuerzos tecnológicos por encontrar nuevas formas para superar la lectura analógica, y de ello dan buena muestra las numerosas páginas sobre ediciones digitales que se encuentran en la red, pero el salto al formato es de gran complejidad. De nuevo se plantea otro gran reto. Es imprescindible que el libro digital dé un paso hacia otro tipo de formatos que permitan lecturas múltiples y no se conformen con ser solo una reproducción de libro tradicional.

4. Adecuar la edición desde la interactividad. Desde esta perspectiva es fácil aventurar que el futuro de la edición pasa por conseguir una mayor interactividad en el libro digital. Hasta el momento las Bibliotecas Virtuales Textuales han conseguido dar un paso adelante creando -alrededor de las ediciones- un mayor número de elementos que interactúen con el lector. De esta forma se consigue que una lectura sea sugerente, atractiva y evoque y despierte todo el mundo de los sentidos. No 
obstante, la interactividad tiene un límite -no solo el referido al formato- sino el propiamente derivado de una buena lectura que hace que no sea conveniente interactuar de forma ilimitada hasta perder el hilo conductor del texto. En este sentido, es importante conseguir un equilibrio entre las aportaciones hechas desde la interacción y la correcta lectura del texto. La labor del editor es el punto clave para conseguir una edición digital de calidad que permita una sana lectura con todos los aportes tecnológicos sin que se desvirtúe el sentido genuino del texto. De nuevo el horizonte pasa por un encuentro entre tradición y futuro que no desestime -entre otras cosas- una interfaz cordial y amable del texto sobre el que se pueda interactuar.

5. Unificar los criterios de acuerdo a la finalidad. Cualquier editor guarda en su haber un ideal. Parece bastante común que muchos sueñen con una utopía: unificar los criterios de edición de acuerdo a los distintos formatos que van surgiendo con los avances tecnológicos. La edición digital se presenta como una gran oportunidad para superar un problema de antaño, que si bien no supuso un problema en la lectura tradicional, sí es una amenaza para una lectura interactiva donde el lector se dirija especialmente a examinar las distintas sugerencias y donde la disparidad de criterios supondría una ruptura o "ruido" entre el escritor y el lector. Hasta el momento los criterios de edición son -como todos sabemos- dispares y múltiples. Existen tantos como ramas del saber, editoriales o revistas especializadas. No es posible encontrarlos organizados al menos por especialidades. Aunque lentamente se avanza hacia formas de citación y criterios de edición cada vez más generalizados, queda mucho por hacer y la edición digital ofrece una oportunidad única para acelerar este proceso de globalización que impone la red.

6. Las nuevas lenguas de marcado. Este apartado constituye otro de los pilares básicos de la edición electrónica. Editar un texto pasa por anotarlo, por permitir que el lector interactúe de forma más fluida. La anotación lleva de la mano al lector hacia una interpretación, y especialmente le dirige para que construya su propia lectura. En la edición digital la anotación tradicional es sustituida por un lenguaje de marcación HTML, XTML, TEI, que impone al editor unos parámetros precisos sobre 
los que incidir. El avance en los tres últimos años ha sido notable y el lenguaje TEI ha intentado incorporar otras perspectivas no tecnológicas y sí del ámbito textual. Sin embargo, aunque este ha sido una gran evolución, sigue siendo necesario profundizar en las lenguas de marcación con el fin de que contemplen, desde la interactividad, las peculiaridades propias de cada texto. Ante este reto se plantean muchas cuestiones, entre otras, ¿podrán dar las lenguas de marcado un salto de las bibliotecas virtuales a los libros electrónicos de manejo habitual?

En definitiva, podríamos resumir que el futuro del libro electrónico está asegurado, pero no su calidad. Dependerá de todos los implicados en este proyecto de futuro, que se generen encuentros $-\mathrm{y}$ no desencuentros- entre los diversos ámbitos. El diálogo entre todos y la plasmación de sus consecuencias, será el mejor aval del libro electrónico y para las diversas formas de edición electrónica. Un proyecto ambicioso e ilusionante, no por eso menos real y posible.

\section{DIGITAL AND ELECTRONIC EDITING IN SPAIN: A STATE OF THE ART}

ABSTRACT: This paper offers an overall perspective on the challenges that the book publishing industry in Spain must face in the near future to fit its current content offer to the new expectations raised by reader-users in the digital era. Starting from some new understanding of the concept of textuality sponsored by the use of new reading devices, this paper highlights the new perspectives that future digital critical editions should bring to provide new contents to a specialized audience in demand for more interaction and content support. Additionally, it emphasizes the importance of helping professionals in the field of text edition, philology and data-processing reach a consensus to surmount the current chaos in the field of digital critical editions.

KEYWORDS: Digital critical editions. Virtual libraries. Markup languages. Hypertext. reader-user.

\section{Referencias}

AAVV. La gran transformación: panorama del sector del libro en España (20122015). Madrid: Anatomía de Red S.L., 2012.

APOLLON, Daniel; BÉLISLE, Claire; RÉGNIER, Phillipe. (Eds.). Digital Critical Editions. Exploring the Interweaving of Traditional and Digital Textual Scholarship. Urbana-Champaign, Chicago and Springfield: University of Illinois Press, 2014. 
LUCÍA MEGÍAS, José Manuel. Hacia nuevos paradigmas textuales (Edición y difusión de los textos literarios en el siglo XXI). En ROMERO LÓPEZ, Dolores; SANZ CABRERIZO, Amelia (Eds.). Literaturas del texto al hipermedia. Barcelona: Antrophos, 2008a. p. 100-115.

LUCÍA MEGÍAS, José Manuel. El hipertexto ante el reto de los textos medievales: nuevas reflexiones sobre informática humanística. En GONZÁLEZ, Aurelio; von der WALDE, Lilia; COMPANY, Concepción (Eds.). Temas, motivos y contextos medievales. El Colegio de México, Universidad Autónoma de México, Universidad Metropolitana, 2008b. p. 425-452.

LUCÍA MEGÍAS, José Manuel. La edición crítica hipertextual: la superación del incunable del hipertexto. Lecturas y textos en el siglo XXI. Nuevos caminos en la Edición Textual. Vigo: Axac, 2009. p. 11-74.

LUCÍA MEGÍAS, José Manuel. Reflexiones en torno a las plataformas de edición digital: el ejemplo de La Celestina. En PAOLINI, Devid (Coord.). De ninguna cosa es alegre posesión sin compañía: estudios celestinescos y medievales en honor del profesor Joseph Thomas Snow. Nueva York: The Hispanic Seminary of Medieval Studies, vol. 1, 2010. p. 226-251.

PAJARES TOSCA, Susana. Literatura digital. El paradigma hipertextual. Cáceres: Universidad de Extremadura, 2004.

PEIRÓ SEMPERE, Julio; JIMÉNEZ FERNÁNDEZ, Concepción M.; ROSA RIVERO, Álvaro; MARTÍNEZ NIETO, Roxana B.; ROLDÁN MARTíNEZ, Ignacio. Detection and Evaluation of the Reading Habits Shown by University Students in the Field if Literary Works on Electronic Media: A Case Study at the International University of La Rioja. IATED INTED2014 Proceedings, p. 6464-6469, 2014.

PIPER, Andrew. Book was there: Reading in Electronic Times. Chicago: The University of Chicago Press, 2012.

PRICE, Kenneth; SIEMENS, Ray. Introduction. Literary Studies in The Digital Age: An Evolving Anthology. MLA, 2013. Disponible en: https://dlsanthology.commons.mla.org/

RODRÍGUEZ DE LAS HERAS, Antonio. El libro al otro lado de la pantalla. En ALONSO Andoni; BLANCO José Pablo (Coords.) Pensamiento Digit@। Humanidades y Tecnología de la Información. Cáceres: Junta de Extremadura, 2001. p. 134-138.

ROJAS CASTRO, Antonio. El mapa y el territorio. Una aproximación históricobibliográfica a la emergencia de las Humanidades Digitales en España. Caracteres. Estudios culturales y críticos de la esfera digital, n. 2, v. 2, p. 10-53, noviembre de 2013. Disponible en: http://revistacaracteres.net/revista/vol2n2noviembre2013/elmapa-y-el-territorio 
Texto recebido em: 13/07/2015.

Texto aceito em: 22/07/2015. 